\title{
Chlamydia pneumoniae and Helicobacter pylori IgG seropositivities are not predictors of osteoporosis-associated bone loss: a prospective cohort study
}

\author{
Mohammad Reza Kalantarhormozi ${ }^{1} \cdot$ Majid Assadi $^{2} \cdot$ Katayoun Vahdat $^{3} \cdot$ \\ Kamyar Asadipooya ${ }^{4}$ - Afshin Ostovar ${ }^{3}$ - Katayoun Raissi ${ }^{1}$ - Hossein Darabi ${ }^{3}$. \\ Shokrollah Farrokhi ${ }^{3}$ - Sina Dobaradaran ${ }^{3} \cdot$ Maryam Farrokhnia $^{5} \cdot$ Iraj Nabipour $^{5,6}$
}

Received: 19 May 2014 / Accepted: 9 May 2015 / Published online: 9 June 2015

(c) The Japanese Society for Bone and Mineral Research and Springer Japan 2015

\begin{abstract}
The potential link between infection with Chlamydia pneumoniae or Helicobacter pylori and osteoporosis has not been investigated in population-based longitudinal studies. A total of 250 healthy postmenopausal women who participated in a prospective cohort study were evaluated for IgG antibodies directed against $C$. pneumoniae and H. pylori, osteoprotegerin (OPG), the receptor activator of nuclear factor kappa B ligand (RANKL), CrossLaps, and osteocalcin. Bone mineral density (BMD) was measured at the femoral neck and lumbar spine at baseline and at follow-up 5.8 years later. There were no significant differences in age-adjusted bone turnover markers, OPG, RANKL, the RANKL/OPG ratio, and BMD between the $C$. pneumoniae and $H$. pylori $\mathrm{IgG}$ seropositive and seronegative subjects $(P>0.05)$. Neither $C$. pneumoniae nor $H$. pylori $\mathrm{IgG}$ seropositivity was associated with age-and body mass
\end{abstract}

Iraj Nabipour

inabipour@gmail.com

1 Department of Endocrine and Metabolic Diseases, The Persian Gulf Tropical Medicine Research Centre, Bushehr University of Medical Sciences, Bushehr, Iran

2 The Persian Gulf Nuclear Medicine Research Centre, Bushehr University of Medical Sciences, Bushehr, Iran

3 Department of Infectious Diseases, The Persian Gulf Tropical Medicine Research Centre, Bushehr University of Medical Sciences, Bushehr, Iran

4 Division of Endocrinology and Metabolism, University of Kansas Medical Center, Kansas City, KS, USA

5 Department of Biochemistry, The Persian Gulf Marine Biotechnology Research Centre, Bushehr University of Medical Sciences, Bushehr, Iran

6 The Persian Gulf Tropical Medicine Research Center, Boostan 19 Alley, Imam Khomeini St, 7514763448 Bushehr, Iran index-adjusted BMD at the femoral neck and lumbar spine or bone loss at the 5.8-year follow-up. In logistic regression analysis, neither $C$. pneumoniae nor $H$. pylori IgG seropositivities predicted incident lumbar or spine osteoporosis 5.8 years later. In conclusion, neither $C$. pneumoniae nor H. pylori IgG seropositivity was associated with bone turnover markers, the RANKL/OPG ratio, BMD, or bone loss in postmenopausal women. In addition, chronic infection with $C$. pneumoniae or $H$. pylori did not predict incident osteoporosis among this group of women.

Keywords Chlamydia pneumoniae $\cdot$ Helicobacter pylori $\cdot$ Bone mineral density $\cdot$ Osteoporosis

\section{Introduction}

Chlamydia pneumoniae is an obligate intracellular human respiratory pathogen that contributes to a wide spectrum of clinical presentations, including atherosclerosis [1]. This bacterium is able to survive in host cells and can affect chronic processes, such as atherosclerosis, through augmentation of the inflammatory system, signaling pathways, and oxidative stress [2]. Thus, it is plausible to consider a contributory role for $C$. pneumoniae infection in accelerated bone loss. Indeed, in vitro and in vivo studies have shown that infection with $C$. pneumoniae produces potentially inflammatory and bone resorptive cytokines and chemokines [3, 4]. Very recently, it has been reported that chlamydial DNA was found in osteoporotic bone tissue [5].

The association of Helicobacter pylori seropositivity with a variety of extradigestive manifestations, such as cardiovascular, immunological, and various other pathologies, has been suggested [6]. However, the link between $H$. pylori and these extradigestive manifestations is 